\title{
La alfabetización mediática e informacional en la era del capitalismo de vigilancia
}

\author{
Media and information literacy in the age of surveillance capitalism
}

\section{Andrés Riva Casas*}

\footnotetext{
* Periodista. Licenciado en Estudios Internacionales (Universidad ORT Uruguay). Docente de Sistema Internacional Contemporáneo en la Licenciatura de Estudios Internacionales en la Universidad ORT Uruguay, donde además dicta un curso sobre Posverdad, Periodismo y Política. $\bowtie$ a.rivacasas@gmail.com https://orcid.org/0000-00030629-3863
}

RECIBIDO: 30.9.2019

ACEPTADO: 21.11 .2019

\section{Resumen}

En todo el mundo, los sistemas democráticos enfrentan un cambio radical en las formas de ejercer la ciudadanía. Esto se explica por el cambio tecnológico y, principalmente, por la emergencia de un nuevo espacio público virtual en el que las reglas de juego son completamente distintas. A partir del término capitalismo de vigilancia, el presente trabajo pretende llamar la atención sobre las amenazas y los desafíos que presenta el cambio tecnológico, al tiempo que proponer un abordaje desde el sistema educativo, integrando la alfabetización mediática e informacional como elemento de formación cívica para el ejercicio de los derechos y las libertades en el nuevo espacio virtual.

Palabras clave: alfabetización informacional, educación sobre medios de comunicación, democracia.

\section{Abstract}

All over the world, democratic systems are facing the radical change of the new ways of citizenship. This is explained by technological advancements and, mainly, by the emergence of a new virtual public space in which game rules are completely different. By introducing the concept of surveillance capitalism, this paper seeks to draw attention to the threats and challenges presented by technological change, while proposing an approach from the educational system, integrating media and information literacy as a civic training to exercise citizens' rights and freedoms in the new virtual space. 
Keywords: alfabetización informacional, educación sobre medios de comunicación, democracia

\section{Introducción}

La democracia, como sistema que distribuye en forma igualitaria el poder político entre los ciudadanos de una misma nación, depende del involucramiento y la participación de estos para asegurar su sustentabilidad (Bobbio, 1994). Las formas de participación han cambiado a lo largo de la historia, pero han tenido como eje el ejercicio del voto y del debate público en los ámbitos comunes de convivencia (Rawls, 1995). La democracia ha dependido del espacio público para desarrollarse y sería impensable si la ciudadanía no fuese capaz de ejercer sus libertades básicas en él. Las libertades de pensamiento, expresión y asociación son esenciales para el ejercicio de la ciudadanía, pero a estos efectos también es fundamental el derecho a la información y a la educación básica, en cuanto el propio sistema se debe a sí mismo la formación de ciudadanos virtuosos (Bobbio, 1986) que formen parte de una misma cultura y defiendan a través de ella una serie de valores y principios que exceden ampliamente los aspectos procedimentales de una democracia.

Naturalmente, la democracia prevé formas de participación ciudadana. Algunas de ellas quedan libradas a la voluntad individual, pero otras han sido establecidas de forma obligatoria, como es el caso del sufragio en el Uruguay.

No obstante, el advenimiento de una nueva era digital supone la introducción de cambios sustanciales en las formas clásicas de participación ciudadana, especialmente en lo que respecta a la estructura y regulación del espacio público.

El cambio es sistémico y afecta simultáneamente varios aspectos centrales de la vida democrática: a) cambia la forma de participar en el debate público, de intercambiar ideas y procesar las diferencias; b) cambia la manera en que las personas se informan y toman conocimiento de la realidad que las rodea, y c) cambian también las formas de involucrarse en la vida democrática.

Comúnmente, sobre todo en lo que respecta al consumo de noticias, estos cambios trascendentales son considerados como un cambio de formato. Pero este concepto, que refiere al cambio de los medios físicos por los digitales y al abandono de los medios tradicionales, como la televisión y el periódico, no es suficiente para dar cuenta de la verdadera transformación que se encuentra detrás. A lo largo del presente trabajo se sostendrá que las nuevas formas de ejercer la ciudadanía han transformado el modo en que las personas conviven en el espacio público y participan del intercambio de ideas. 
Los principales aspectos de este cambio son: a) la transformación del sistema capitalista, en el que los ciudadanos han modificado el lugar que ocupan, y b) el surgimiento de un nuevo espacio público virtual con características propias que guarda grandes diferencias con el espacio público en el que se desarrollaron las sociedades de masas.

Este ensayo pretende llamar la atención sobre los riesgos que presenta para el ejercicio de la ciudadanía la irrupción de un nuevo espacio público virtual cuya estructura se erige en ostensible desconexión con aquella que regula al espacio público físico o tradicional.

Se introducirá, como fundamentación teórica de esta disociación del espacio público, el concepto de capitalismo de vigilancia, que supone una reestructuración profunda de las relaciones de poder en las sociedades contemporáneas. Asimismo, este trabajo se propone defender la idea de que, si se quiere evitar regulaciones excesivas que tengan un efecto restrictivo sobre las libertades fundamentales, la mejor alternativa para combatir los efectos adversos del cambio tecnológico consiste en una reformulación de las políticas públicas que desde la educación se propongan formar a los futuros y actuales ciudadanos para nuevos modos de ejercer la ciudadanía en el espacio público virtual.

\section{El acceso a la información como problema global}

La alfabetización mediática e informacional (AMI) es un fenómeno de creciente impacto global, especialmente desde que las campañas de desinformación han demostrado su potencial para desestabilizar procesos democráticos. Esas campañas representan hoy una de las preocupaciones centrales dentro de los regímenes democráticos. Para algunos regímenes autoritarios, la capacidad de interferir en la política de sus adversarios ideológicos se ha tornado una actividad constante, y esta situación ha puesto a las democracias en una encrucijada: la de proteger sus instituciones y valores sin atentar contra las libertades básicas (Walker, 2018).

El puntapié inicial de la AMI podemos encontrarlo en el año 2007, cuando la Conferencia General de la UNESCO hizo un llamado para explorar nuevas formas de apoyar y reforzar las prácticas «con el objetivo general de otorgar a los usuarios la oportunidad de realizar juicios informados sobre las fuentes mediáticas y de información y de ampliar su participación cívica en los medios de comunicación», lo que implica el uso ético de los medios de información y la tecnología para un acercamiento crítico a los cambios sociales (Wilson, 2012).

En este marco global, muchos países han apelado a reformas y modificaciones del sistema educativo, aprobando leyes y financiando programas para educar a los futuros 
ciudadanos a fin de que tengan una mirada crítica del espacio público virtual y sus características.

De acuerdo con un relevamiento de experiencias internacionales realizado para este trabajo, en Estados Unidos hay 13 gobiernos estatales que han aprobado y llevan a cabo políticas públicas de AMI: California, Colorado, Connecticut, Florida, Illinois, Massachusetts, Minnesota, New Jersey, New Mexico, Ohio, Rhode Island, Utah y Washington. La mayor parte de las leyes se han aprobado entre 2017 y 2019, fecha coincidente con los dos mayores eventos concientizadores sobre esta materia: el Brexit y las elecciones norteamericanas de 2016 en las que Donald Trump se convirtió en presidente (Deibert, 2019).

En Europa, las campañas de desinformación y los discursos de instigación al odio han generado alarma en muchos países que también han adoptado medidas para reforzar la respuesta educativa ante estos fenómenos. Son varios los países europeos que evidencian el avance de la AMI: Finlandia, Holanda, Suecia, Estonia, Irlanda, Inglaterra y Portugal ya cuentan en sus currículos escolares con programas destinados formar a los ciudadanos para navegar en el nuevo espacio virtual.

La AMI supone una evolución necesaria de las políticas públicas de formación ciudadana adaptada a las nuevas formas de participar e involucrarse en el debate público, de ejercer los derechos y libertades. Sin embargo, también se ha convertido en una reacción a los efectos adversos de un nuevo espacio público virtual que prolifera, con estructuras opacas e intereses ocultos, al margen de toda regulación nacional e internacional.

No se trata, por tanto, del fenómeno superficial de las noticias falsas o fake news, sino de un problema más complejo y profundo que tiene que ver con la forma en que la información circula, es recibida y evaluada por los usuarios y, finalmente, compartida o retransmitida.

Este abordaje no solo pretende llamar la atención sobre un fenómeno global que muy tímidamente se ha comenzado a debatir en nuestro país. En un contexto en el que la academia y el sistema político coinciden mayoritariamente en la necesidad de llevar a cabo una reforma del sistema educativo, se torna indispensable incluir a la AMI como una dimensión fundamental de las políticas educativas para la formación de ciudadanos que convivan con los riesgos de la era digital.

Un ejemplo elocuente de esta realidad es la forma en que la organización Eduy 21, piedra angular del debate sobre la reforma educativa en Uruguay, ha obviado todos los temas referidos a la AMI o directamente a la formación para el ejercicio de la ciudadanía digital. De hecho, si se hacen búsquedas por palabras o conceptos en su Libro abierto, solo se encuentran referencias puntuales a las tecnologías digitales o la existencia de mundos virtuales, pero ningún abordaje específico (Eduy21, 2018). 
Como veremos a continuación, obviar el impacto del mundo virtual sobre el mundo físico y desatender la brecha existente entre el espacio público físico y el virtual puede suponer un riesgo creciente para las democracias.

\section{Una nueva fase del capitalismo}

El mayor impacto de la nueva era digital es que ha modificado los términos en que se desarrollan las relaciones económicas y de poder en las sociedades abiertas. Es incuestionable que el impacto de la tecnología trasciende a las sociedades democráticas, pero en aquellas sociedades abiertas, que cultivan la libertad y el pluralismo, las nuevas tecnologías de la información plantean un riesgo inédito. La libertad ha siendo históricamente un arma de doble filo para la democracia, porque en ella conviven visiones plurales y en ocasiones antagónicas. La desinformación siempre ha existido, pero con la irrupción de una nueva era digital lo novedoso es la velocidad con que circula, la opacidad de los mecanismos que la distribuyen, la infraestructura tecnológica que se pone a su servicio y los enormes vacíos legales en los cuales se ampara (Presily, 2017). Los impactos del cambio tecnológico en esta era digital operan a todo nivel y debemos estar preparados para nuevos efectos y externalidades, especialmente porque la interacción entre el mundo virtual y el mundo físico es una realidad que ha llegado para quedarse (Schwab, 2016).

El informe 2019 de Freedom House sobre libertad en internet concluye que las plataformas de redes sociales se han convertido en el nuevo campo de batalla para las democracias y destaca que, en el último año, 24 países vieron sus procesos electorales intervenidos mediante estrategias de desinformación:

Shaping the flow of information on the internet is now an essential strategy of those seeking to disrupt the democratic transfer of power through elections. (Shabhaz y Funk, 2019)

Inicialmente, la lógica abierta y horizontal de internet despertó el optimismo sobre una democratización saludable de la generación de contenidos en línea, una ampliación formidable de las posibilidades de obtener información y emitir opiniones, además de convertirse en una potencial herramienta de control contra los abusos de la autoridad (Periser, 2011; O’Neil, 2016). Sin embargo, esas promesas de democratización y ensanchamiento de los espacios de libertad quedaron rápidamente en el olvido (Deibert, 2019). La primavera árabe de 2011 fue una clara señal de la potencialidad y el riesgo implícito en el funcionamiento de las nuevas tecnologías, que empoderaban al ciudadano al mismo tiempo que minaban su camino hacia la libertad (Brown, 2013; Youmans 
y York, 2012). En pocos años internet pasó de ser la panacea de la democracia a convertirse en su peor enemiga (Mounk, 2016).

Las nuevas relaciones de poder que surgen dentro de las sociedades contemporáneas a raíz del desarrollo de una nueva era digital, del internet 2.0 y de la cuarta revolución industrial ya no pueden ser analizadas con los parámetros previos de una democracia sustentada en un capitalismo industrial y financiero.

Ese es el poderoso argumento que Shoshana Zuboff (2015) ha desarrollado en torno al término capitalismo de vigilancia, para dar cuenta de una nueva fase del capitalismo gestada en paralelo con las nuevas tecnologías de la información y la comunicación y su empleo en tareas de vigilancia. Después de los atentados del 11 de setiembre de 2001, la vigilancia emergió como un concepto estrictamente vinculado a la seguridad, un punto de inflexión en las políticas de seguridad global y un incentivo para el desarrollo -y la aceptación pública- de nuevas y más sofisticadas formas de vigilancia y control social (Lyon, 2018).

Pero, como expresa David Lyon, la vigilancia se ha convertido en un modo de vida desde que las nuevas tecnologías nos permiten observarnos constantemente los unos a los otros (Lyon, 2018). Esto ha generado una cultura de la vigilancia que invita a las personas - más allá del rol de gobiernos y corporaciones - a involucrarse crecientemente en prácticas de vigilancia sobre sus pares (Lyon, 2018).

Al mismo tiempo, los ciudadanos que viven bajo constante vigilancia suelen ignorar la magnitud del problema. Por un lado están los que ignoran verdaderamente las implicaciones de una cultura de la vigilancia. Por otro, los que prefieren creer en que esa cultura es necesaria y beneficiosa, al tiempo que se aferran a la idea de que ellos no saldrán perjudicados o de que, si nada tienen que ocultar, tampoco deben preocuparse (Bauman y Lyon, 2013).

Monhan (2011), por su parte, nota que las prácticas de vigilancia pueden dividirse en tareas de cuidado y de control. En otras palabras, podemos identificar en ellas objetivos de protección y seguridad al mismo tiempo que intenciones de controlar el comportamiento de las personas con fines de seguridad, políticos o comerciales. Según su definición, la vigilancia consiste en el monitoreo sistemático de personas o grupos de personas con el objetivo de regular y gobernar su comportamiento.

La cultura de la vigilancia ha alterado el funcionamiento de las sociedades democráticas. Según Zuboff (2015), el cambio es radical y requiere nuevos enfoques desde el punto de vista sociocultural, económico y político. El capitalismo de vigilancia, dice la autora, consiste en una nueva lógica de acumulación que establece nuevas relaciones políticas y sociales que sustituyen los contratos, el imperio de la ley y la confianza a través de la imposición de una nueva forma de soberanía que se mueve en la opacidad.

En su reciente libro Surveillance Capitalism, publicado en 2019, Zuboff profundiza las raíces históricas de este fenómeno y ofrece una definición más compleja y acabada: 
Surveillance capitalism unilaterally claims human experience as free raw material for translation into behavioral data. Although some of these data are applied to product or service improvement, the rest are declared as a proprietary behavioral surplus, fed into advanced manufacturing processes known as 'machine intelligence', and fabricated into prediction products that anticipate what you will do now, soon, and later.

Luciano Floridi (2019) ofrece una versión histórica similar a la de Zuboff al hacer referencia a la forma en que el poder - gris, según su descripción- se traslada desde la producción de manufacturas hacia la producción y acumulación «de información sobre las personas y las cosas».

El capitalismo de vigilancia implica varias modificaciones en lógica del funcionamiento social que reconfiguran el rol del ciudadano, alteran la forma en que este interactúa con otros y, muy especialmente, modifican los resultados de la interacción social en el espacio público virtual y físico.

Sus características centrales son al menos cinco: a) utiliza los datos personales y privados de los individuos como nueva materia prima para extender sus dominios; b) esos datos le permiten crear un nuevo mercado de predicción y modificación del comportamiento humano que es la mayor fuente de ingresos de las empresas; c) las prácticas de extracción y utilización de esos datos erosiona la confianza en la sociedad y altera la naturaleza de los contratos; d) este nuevo escenario altera el concepto de autoridad generando nuevas asimetrías de poder, y e) encuentra en la democracia un obstáculo para su crecimiento y futuro desarrollo.

a. El capitalismo de vigilancia utiliza los datos personales de los usuarios virtuales como materia prima. A través de su acumulación, procesamiento y análisis, los paquetes de datos pueden ser vendidos como un elemento predictivo del comportamiento humano. Esa necesidad de grandes volúmenes de datos establece una lógica acumulativa que es central en esta nueva fase del capitalismo. "The logic of accumulation produces its own social relations and with that its conceptions and uses of authority and power», dice Zuboff (2015).

La lógica acumulativa se expresa a través del término big data, que según la autora es al mismo tiempo una condición y un resultado del capitalismo de vigilancia. Gobiernos y empresas, a través de diversas formas y estrategias de vigilancia, han adquirido la capacidad recabar y analizar enormes volúmenes de datos.

Por otra parte, los datos privados que se acumulan no son robados, sino generados cotidianamente por los usuarios mientras navegan en internet. Esa información privada, cuyo almacenamiento y posterior utilización el ciudadano desconoce, genera una estela de datos que luego son utilizados en su contra. «Populations are the sources from 
which data extraction proceeds and the ultimate targets of the utilities such data produce», dice Zuboff (2015).

Según expresa Cristóbal Cobo: «[...] hoy vivimos en una economía de los datos basada en un sofisticado aparato de vigilancia de extraordinario alcance, que acapara casi cualquier aspecto de la información personal» (Cobo, 2019b, p. 28). O’Neil (2016) advierte que la información personal (registros médicos, judiciales, policiales, etcétera) está siendo usada por algoritmos automatizados para definir el futuro de un trabajador, el monto de la póliza de seguros de un ciudadano o sus probabilidades de reincidir si ha cometido un delito. Y en el plano político, para incidir directamente sobre las definiciones políticas de los individuos (Persily, 2017).

La abundancia de información que se genera a la sombra de la vigilancia habilita la existencia de un modelo de negocios que se sustenta en la segmentación específica del público. Se desarrollan estrategias de marketing individualizadas a la medida de cada usuario que se tornan alarmantes cuando los algoritmos conocen, incluso mejor que nosotros, aquello que pensamos y sentimos (Helbing et al., 2017).

Esta nueva versión del capitalismo ha logrado convertir en ingresos la vida diaria de las personas, sus hábitos y emociones, rutinas y relaciones. De tal forma, al ofrecer servicios gratuitos, las compañías de internet se nutren infinitamente de la información que los usuarios generan.

Vale destacar, a su vez, que el capitalismo de vigilancia tiene la limitación de proyectar una visión esencialmente pesimista de la relación que se establece entre la democracia y la tecnología en la era de la cuarta revolución industrial. Como han demostrado Richard Thaler y Cass Sunstein (2008), la utilización del big data puede permitir a los gobiernos ayudar a sus ciudadanos a tomar mejores decisiones en aquellos aspectos de su vida en los que los sesgos cognitivos les impiden actuar de forma racional. Ambos autores han acuñado en torno a esta idea el término paternalismo libertario (Thaler y Sunstein, 2003) y han defendido el rol de los arquitectos de la decisión; aquellos que con el poder de la información son capaces de influenciar —desde un punto de vista positivo - las decisiones que toman las personas en su vida diaria.

b. Como ya se ha mencionado, el potencial que ofrece el análisis de los datos es la predicción y modificación del comportamiento humano (Zuboff, 2019; Monhan, 2011; Lyon, 2018). El poder, en esta nueva etapa, no radica en la propiedad de los medios de producción, sino en el control de los elementos que permiten predecir y modificar el comportamiento humano. De esta forma, las compañías son capaces de orientar las decisiones de cada usuario, imprimiendo sutiles modificaciones en su forma de percibir la realidad, en su estabilidad emocional o en la forma en que se vincula con el entorno.

Esta dinámica ha sido descrita también mediante el término economía de la atención, por la forma en que las empresas obtienen ganancias de los usuarios pegados a las pantallas de sus dispositivos inteligentes (Peirano, 2019). 
Los desarrollos más evidentes en esta área se han realizado en el área empresarial; sin embargo, el caso de la consultora británica Cambridge Analytica evidencia el impacto real de la recolección y el análisis de datos sobre los procesos electorales. La exdirectora de desarrollo de negocios de la empresa, Brittany Kaiser (2019), explica en su reciente libro cómo se utilizaron los datos de Facebook de millones de usuarios en Estados Unidos para elaborar perfiles psicográficos y luego enviarles mensajes políticos personalizados para influir en su voto. Este método fue el empleado durante la campaña de Donald Trump para ganar las elecciones en Estados Unidos en el 2016. A su vez, en el Reino Unido, la evidencia presentada por Kaiser ante una de las comisiones del Parlamento británico prueba la colaboración que existió entre Cambridge Analytica, el grupo Leave.EU y el Partido de la Independencia del Reino Unido (UKIP) para ganar el referéndum (Digital, Culture, Media and Sports Committee, 2019).

De esta forma, la lógica de la era digital supone un riesgo claro para los procesos electorales que dependen de una sociedad civil fuerte y de debates públicos informados.

c. Según expresa Zuboff (2015), la nueva lógica del capitalismo de vigilancia supone además una reconfiguración del contractualismo que fue piedra angular para el desarrollo de la democracia moderna. Peirano (2019) considera que la aceptación automática e irreflexiva de los términos de uso genera una rutina similar a un hábito o incluso una adicción a través del cual las personas aceptan, sin leer, un contrato legal vinculante en el que los usuarios renuncian a sus derechos en favor de las compañías que recopilan sus datos.

Para Zuboff (2015), los términos de uso erosionan la relación histórica entre los mercados y las democracias, en tanto el capitalismo de vigilancia es inmune a las reciprocidades típicas del capitalismo, en el que el capitalista y la población se necesitaban mutuamente para la generación de empleo y consumo. Los nuevos contratos de la era digital, dice Zuboff (2019), imponen condiciones de tómalo o déjalo que se convierten en una forma opresiva de aceptar condiciones, dado que nadie, o casi nadie, se dedica a leer los términos y condiciones que aplican.

A su vez, ese nuevo estatus de los contratos como un subproducto de la interacción entre el ciudadano y una máquina elimina a la confianza como factor de cohesión.

d. La alteración del sistema capitalista y de sus fundamentos, expresa Zuboff (2019), supone un cambio en las relaciones de poder que resulta en una mutación del concepto de autoridad. La autora describe el nacimiento de una «nueva clase de mano invisible» capaz de administrar a su gusto las relaciones de autoridad y poder. En ese sentido, la autoridad es sustituida por la técnica, que establece un sistema impersonal de disciplina y control que produce conocimiento sobre el comportamiento humano sin consentimiento de las personas (Zuboff, 2015).

Apelando a la definición marxista del concepto de ideología, Zuboff (2015) considera que esta nueva forma de ejercer el poder y la autoridad se resumen en la capacidad 
de crear una falsa conciencia a través de los hechos ocultos y la comercialización del comportamiento. Hay influencia y manipulación, pero no coacción física.

Al mismo tiempo que mutan los conceptos de autoridad y poder, también se modifican las formas de distribución de este último. En el marco del capitalismo de vigilancia y la economía de la atención, las asimetrías en conocimiento o posesión de información se convierten inmediatamente en asimetrías de poder. Como advertía Alvin Toffler (1992, p. 120): «Dado que reduce la necesidad de materias primas, trabajo, tiempo, espacio y capital, el conocimiento pasa a ser el recurso central de la economía avanzada». El autor notaba, al mismo tiempo, que uno de los componentes del cambio de poder radicaba en el control de las autopistas informáticas: "A medida que se profundiza esta histórica fusión de telecomunicaciones y finanzas, el poder inherente en el control de las redes aumenta exponencialmente» (1992, p. 143).

Toffler advertía, además, sobre el riesgo de opacidad inherente a un futuro en el que las redes fuesen inteligentes y automatizadas, porque esto traería como consecuencia procesos ocultos a la vista humana y una mayor dependencia respecto a acontecimientos preprogramados que «pocas personas comprenden» (1992, p. 161). El autor predecía, además, un futuro en el que el poder radicaría «en el conocimiento acerca del conocimiento». En ese sentido, Zuboff (2019) explica que el capitalismo de vigilancia se sustenta en las asimetrías de información que existe entre los ciudadanos y aquellos que controlan los medios de modificación del comportamiento.

Richards (2013) añade que la vigilancia afecta el balance de poder entre los individuos y aquellos que desarrollan las prácticas de vigilancia, por cuanto incrementa el riesgo de persuasión, chantaje y otros usos perjudiciales de la información sensible que es recabada. La vigilancia siempre conlleva el riesgo de convertirse en dominación, es decir, que la vigilancia nace como una amenaza constante a la supresión de las libertades. Desde que la vigilancia y la sistematización de la información le confieren al que observa suficiente poder para influir en el comportamiento de sus víctimas, la vigilancia cobra un carácter sombrío y antidemocrático.

Por último, la vigilancia permite a los dueños de la información clasificar a las personas según diferentes parámetros. Cuando las personas quedan a merced de algoritmos diseñados gracias a las prácticas de vigilancia y procesamiento de datos, la discriminación se convierte rápidamente en factor inherente a la democracia. Los algoritmos, confeccionados por humanos, suelen reproducir muchos de los prejuicios de sus autores o crear nuevos con base en errores metodológicos. Como afirma Richards (2013), «the power of sorting can bleed imperceptibly into the power of discrimination».

Finalmente, el capitalismo de vigilancia rompe con la lógica según la cual el capitalismo requiere de la democracia para asegurar su prosperidad. Este sistema político, con sus garantías, pesos y contrapesos, aseguró por décadas al sector privado la capacidad de desarrollar empresas con un horizonte de estabilidad y respeto por el Estado de 
derecho, la legalidad de los contratos, etcétera. Pero para el capitalismo de vigilancia la democracia ya no es un medio para la prosperidad, sino que se ha convertido en una amenaza a las ganancias que el mercado promete (Zuboff, 2015).

Cristóbal Cobo lo resume de manera muy simple: «Todos estos aspectos hacen que sea necesario crear nuevos límites a los datos que las empresas recopilan, además de un mayor control para las personas sobre quién, cómo y cuándo se utilizan sus datos personales» (Cobo, 2019b, pp. 28-29). El problema, como apunta Richards (2013), es que nos aproximamos lentamente a un mundo de vigilancia total e irrestricta.

\section{El surgimiento de un nuevo espacio público}

En las sociedades democráticas actuales conviven dos versiones distintas del espacio público cuya interrelación es compleja. Una de ellas es la tradicional, la del mundo físico, que permite encuentros y desencuentros entre los ciudadanos que conforman la esencia fundamental del debate público. La otra, más novedosa y por tanto razonablemente incomprendida, es la que se desprende de la interacción de esos mismos ciudadanos en el mundo virtual, donde las plataformas como Facebook, Twitter, WhatsApp e Instagram, entre otras, proveen las condiciones necesarias para que las personas se conecten, comuniquen y compartan contenidos creados por ellas mismas o por terceras personas.

Entre estos dos espacios, similares y al mismo tiempo profundamente diferentes, lo que cambia son las reglas de juego y, por ende, también los resultados de la participación ciudadana en ellos. En el espacio físico, donde se producen las interacciones del mundo real en términos de Robert Putnam (2000), los ciudadanos deben aprender a lidiar con la diversidad en todos los términos que la expresión admite. Las comunidades deben afrontar la diversidad de ideas políticas, confesiones religiosas, modos de vida, gustos culinarios y toda una gama de variedades y matices que hacen a una sociedad heterogénea. No en vano la democracia liberal se sustenta en el pluralismo y la tolerancia, que son conceptos y al mismo tiempo valores inherentes a la filosofía liberal. Ya a comienzos de siglo, Putnam advertía:

Real-world interactions often force us to deal with diversity, whereas the virtual world may be more homogeneous, not in demographic terms, but in terms of interest and outlook. Place-based communities may be supplanted by interest-based communities. (Putnam, 2000)

Esa homogeneidad del espacio público virtual se ha convertido hoy en una realidad compleja y amenazante, porque, si bien se encuentra aparentemente divorciado del 
espacio público físico, ambos se afectan mutuamente. El surgimiento de un nuevo espacio público no significa necesariamente la muerte del otro, sino la promesa de una convivencia inconexa y distorsionada.

El desafío radica en evitar el desencuentro y la desinformación, porque el espacio público debe ser un espacio para la construcción de acuerdos. Y aunque eso no siempre se consiga, las discusiones diversas en clave democrática contribuyen a la construcción de una identidad tolerante y plural (Papacharissi, 2008).

La democracia necesita experiencias comunes y debates sobre asuntos de interés general para la ciudadanía. El riesgo es inaugurar una lógica de fragmentación social que divida a la sociedad en burbujas enemigas del disenso. Un sistema de comunicaciones que estrangula la existencia de experiencias comunes fomentará la división y la incomprensión (Sunstein, 2016).

La fragmentación social, en este sentido, surge como una aproximación sociológica a lo que Eli Pariser bautizó como filtros burbuja (Pariser, 2011). La gran diferencia del espacio público físico con aquel que se ha desarrollado en el mundo virtual radica en que los intermediarios de ese espacio tienen la capacidad de filtrar la información que recibimos. Si a eso le sumamos el desarrollo cada vez más complejo y sofisticado de algoritmos que filtraran la realidad de manera personalizada, el resultado es un espacio público hecho a la medida de cada ciudadano. Este fenómeno supone un riesgo serio para la democracia porque atomiza y superpone una serie infinita de realidades paralelas que carecen de puntos de encuentro (Pariser, 2011). Y si bien es posible afirmar que la fragmentación geográfica habilita escenarios similares, la novedad aquí es que dos personas, aunque vivan bajo el mismo techo, pueden acceder a realidades completamente distintas e incluso contradictorias.

Bozdag y Van den Hoven (2015) añaden que los filtros burbuja representan un riesgo desde el punto de vista liberal en cuanto limitan la autonomía individual y la capacidad de elegir libremente. Sin acceso a una pluralidad de puntos de vista, los ciudadanos no pueden hacer juicios razonables ni decidir en función de sus mejores intereses.

Hay, en este escenario nuevo para la democracia y desafiante para el ejercicio de la ciudadanía, tres aspectos que no deben ser obviados.

\section{Personalización y atomización del espacio público}

El primer aspecto es que los nuevos intermediarios del espacio público tienen la capacidad de generar escenarios a la medida de los gustos y la personalidad de cada usuario. Como afirma Pariser, los filtros burbuja son invisibles y en ellos, cada uno se encuentra solo y aislado. De esa forma, el usuario será expuesto únicamente a los aspec- 
tos de la realidad que sean de su agrado. Esto adquiere un tenor sórdido cuando es trasladado al plano de las ideas políticas, de las tendencias religiosas o de los simples y cotidianos debates que una sociedad civil robusta debe mantener. Las redes sociales, con el fin de construir para cada usuario un entorno amigable en el que se sienta cómodo, han creado burbujas de aislamiento en las que el ciudadano solo se vincula con personas e ideas que son de su agrado, lo que altera la forma en que se encuentran la información y las ideas (Pariser, 2011).

Estas burbujas suponen un entorno acolchonado, de aristas limadas, en el que el usuario se siente cómodo, pero cuya estructura supone una incógnita. El ciudadano desconoce la forma en que se estructura la nueva realidad, pero se siente cómodo en ella, a tal punto que desiste de cualquier intento de cuestionar los vínculos que crea y a los que está sometido. Las asimetrías de información operan en su contra.

Esto, que no sucedía en el espacio público físico, representa tal vez el mayor desafío de un sistema educativo que pretenda educar para la libertad dentro de la estructura de un régimen democrático: si ha cambiado la forma de procesar las diferencias en el espacio público, entonces debemos preparar a los futuros ciudadanos para el ejercicio de sus derechos y libertades en este nuevo escenario. Y no se trata de prevenir posibles riesgos aislados, sino de educar a las personas para ejercer la ciudadanía en un nuevo entorno, con nuevas reglas, que convive y se superpone con el antiguo formato de espacio público.

El debate amplio e informado es la piedra de toque para el proceso de toma de decisiones a través del cual los ciudadanos definen su voto y sus opiniones. Cuando el modelo de comunicación masiva era de uno para muchos, es decir, cuando existían los intermediarios de interés general que eran los periódicos, las radios y las televisoras, la sociedad tenía acceso a debates comunes. Pero desde el momento en que la comunicación se ha transformado hacia un modelo de muchos para muchos, la creación y distribución de contenidos se ha tornado incontrolable (Mounk, 2018). Paradójicamente, esa democratización ha dado como resultado un espacio público más fragmentado, en el que los puntos en común se hacen borrosos y difíciles de identificar.

El peligro mayor aparece, según Cobo, «cuando dejamos de pensar y permitimos que la toma de decisiones basada en datos influya y juegue un papel clave en nuestra vida personal a la hora de comunicarnos con otros, definir nuestras relaciones digitales o al filtrar la información que queremos consumir» (Cobo, 2019b, p. 28).

\section{Cambio de condiciones en el ejercicio de la ciudadanía}

Otra característica destacable es que las condiciones de ejercicio de la ciudadanía, en el nuevo espacio público, son profundamente diferentes, porque el capitalismo de 
vigilancia los convierte en materia prima. Y la alternativa —es decir, mantenerse al margen-puede ser extremadamente costosa, porque la participación en el mundo virtual forma parte de su identidad y allí se desarrollan conceptos como los de familia y amistad. Como explica Balaguer (2017), la tecnología moldea el concepto de identidad.

Este nuevo espacio público se estructura de una forma muy diferente y sus lógicas de funcionamiento ofrecen resultados muy distintos como consecuencia de las interacciones entre las personas. Todas las garantías para el ejercicio de los derechos y las libertades que la democracia construyó para el espacio público tradicional no necesariamente son válidas para el nuevo espacio virtual. Esta situación ofrece como resultado a un ciudadano que se encuentra a sí mismo desvalido, despojado de las fortalezas que rigen el sistema democrático (Helbing et al., 2017).

En este nuevo formato, la relación de los usuarios con los proveedores de servicios virtuales, como los buscadores y las plataformas de redes sociales, es de una debilidad extrema. La capacidad de filtrado, según los expresa Sunstein (2016), es doble. Por un lado, las personas pueden filtrar lo que ven y aislarse voluntariamente del debate público, mientras que las empresas pueden filtrar a las personas con base en la información que estas han generado y por la cual han sido cuidadosamente categorizadas.

En este sentido, y dado que las reglas que hasta ahora fueron útiles probablemente dejen de serlo en el futuro cercano, urge repensar las formas de ejercer la ciudadanía y educar para su ejercicio. Las señales de alarma están a la vista. En Europa, las tecnologías de la información están siendo utilizadas para expandir y arraigar el discurso populista, que llevado al escenario virtual permite el desarrollo de una versión de la democracia digital como vehículo para reivindicar la democracia directa, dado que las personas tienen capacidad y voluntad para expresarse constantemente en ese otro espacio público (Floridi, 2016).

El gran drama al que nos enfrenta la nueva era digital es que, en las condiciones actuales, el espacio público promete una ciudadanía cada vez menos informada —o más desinformada-, organizada en torno a burbujas de afinidad en las que el disenso se pierde y el debate público se resiente. Esto nos obligará a explorar las ventajas y las limitaciones de la regulación, que tarde o temprano deberá establecer límites claros al funcionamiento del espacio virtual.

\section{Cambio en los patrones de consumo de información}

Según un estudio de Pew Research, el 68 \% de los norteamericanos consumen noticias a través de las redes sociales, aunque sea esporádicamente (Pew Research, 2018).

Según un estudio de la Universidad Católica, en Uruguay el peso de internet como principal fuente de información creció del 40 \% en 2013 al 56 \% en 2017. El 85 \% de los 
uruguayos son usuarios de internet y el $68 \%$ lo utilizan a diario. A su vez, el $82 \%$ de los internautas se conectan diariamente a internet a través del teléfono celular. El estudio indica, además, que $64 \%$ de los internautas utilizan internet todos los días para buscar o leer noticias, y el 37 \% diariamente para confirmar un dato o hecho (Dodel, 2018).

De acuerdo con el estudio Perfil del internauta uruguayo de Radar (2018), el $89 \%$ de los uruguayos son usuarios de internet con una conexión promedio de seis horas por día. Tomando en cuenta los teléfonos inteligentes (45\%), el $97 \%$ de los hogares cuenta con al menos una computadora. A su vez, WhatsApp, Facebook y YouTube figuran entre las aplicaciones más frecuentes en los celulares.

Según un reciente informe del Reuters Institute, la tendencia en las generaciones más jóvenes es muy clara respecto al consumo de noticias. Hoy el $45 \%$ de los jóvenes de 18 a 24 años tienen su primer contacto con la información a través de su teléfono inteligente, mientras que los mayores de 35 lo hacen en un $19 \%$. Más aun, los jóvenes utilizan en un $54 \%$ las redes sociales como principal fuente de noticias, algo que en los mayores desciende a $29 \%$ (Newman, Fletcher, Kalogeropoulos y Nielsen, 2019).

Facebook, que se ha convertido en una plataforma de consumo de información para millones de personas en todo el mundo, no produce sus propias noticias, sino que solamente se dedica a jugar un rol de intermediario entre el ciudadano y la fuente de información.

Sin embargo, ese rol de intermediación no es simple ni tampoco neutral. De acuerdo con su capacidad de filtrado, las plataformas digitales han integrado a la información como parte de esa estrategia. La neutralidad de empresas como Google o Facebook es un mito largamente extendido, lo que afecta la capacidad de los usuarios para comprender de qué manera las plataformas virtuales afectan la calidad de la información (Chander y Krishnamurthy, 2018).

La conformación de burbujas tiene como resultado que las personas consumen la información que las plataformas creen más adecuadas según sus gustos y prejuicios. Por eso, para mesurar el impacto de este nuevo escenario en la vida de las personas, es necesario abandonar la idea de neutralidad que las propias empresas tecnológicas han contribuido a construir.

La salvaguarda de un espacio público diverso requiere que las personas se enfrenten a ideas con las que no están de acuerdo o con informaciones que no necesariamente son de su interés; de lo contrario, ¿cómo podrán tomar decisiones sobre asuntos de interés general? 


\section{Una respuesta educativa}

Los cambios que se han procesado en las últimas décadas a raíz del advenimiento de la nueva era digital y las potencialidades de las nuevas tecnologías nos permiten predecir un impacto más profundo cuanto más tiempo pase sin que se adopten medidas, lo que impone la necesidad de una mirada introspectiva.

La tecnología actual supone la llegada de transformaciones sistemáticas y sucesivas, de carácter radical, capaces de poner en riesgo la propia estructura del sistema democrático. No nos enfrentamos a un cambio superficial, sino a una modificación de las reglas de juego que amenazan con declarar la obsolescencia de las instituciones, las normas y los valores democráticos.

La instauración de un nuevo modelo económico basado en los datos personales, la capacidad creciente para predecir y modificar el comportamiento humano y la disociación del espacio público en dos versiones que transcurren en paralelo y con reglas diferentes son parte central del momento de transformación al que nos enfrentamos y para el cual debemos prepararnos.

La paradoja, para muchos regímenes democráticos, es tomar medidas preventivas para evitar problemas mayores o medidas de contingencia para lidiar con los problemas una vez que se han presentado. La regulación, en este sentido, puede ser aliada o enemiga, según si el foco está puesto en promover la libertad y la autonomía o en la censura del discurso y del intercambio en el espacio público.

Cualquiera sea el caso, no podemos olvidar que al regular el espacio público y a sus intermediarios lo que se pone en juego es la libertad de expresión. Desde esta perspectiva, la regulación del discurso en las redes sociales siempre lleva implícito el riesgo de la censura.

\section{Nueva ciudadanía, ¿nueva educación?}

Durante décadas, el cambio tecnológico y la llegada de una era digital donde la vida puede transcurrir online alimentaron el concepto de nativo digital, cuya aproximación determinista y al mismo tiempo evolutiva de la relación de las nuevas generaciones con la tecnología supuso un abordaje erróneo de sus implicaciones para el ejercicio de la ciudadanía.

Por mucho tiempo, la alfabetización digital fue sinónimo de formación técnica para el uso de las nuevas herramientas, como los ordenadores personales, los teléfonos celulares, procesadores de texto, etcétera. Este abordaje no suponía efecto alguno sobre el funcionamiento de las estructuras o los valores democráticos. 
Según Brown y Czerniewicz (2010), en cambio, el concepto de nativo digital resulta erróneo para evaluar los nuevos desafíos que presenta el ejercicio de la ciudadanía en el espacio público virtual. Los nuevos ciudadanos, por el simple hecho de nacer en este tiempo histórico, no pueden presumirse mejor capacitados para el aprovechamiento de las oportunidades que supone la nueva era digital. De acuerdo con Mossberger (2009), un abordaje que no ponga el foco en la formación para la nueva era digital corre el riesgo de profundizar las brechas y reproducir las formas actuales de desigualdad en el acceso a las oportunidades, o de incrementar las barreras a la participación política igualitaria.

El cambio en las formas de ejercer la ciudadanía nos impulsa a evaluar modificaciones en la formación de los futuros ciudadanos, especialmente en lo que respecta al ejercicio de sus derechos y libertades en un nuevo escenario que mantenga los valores y las instituciones del sistema liberal-democrático. Eso implica, como explican Helbing et al. (2017), conocer la forma en que se compone ese nuevo escenario y las reglas que rigen en él. Es indispensable conocer cómo el mundo digital se interrelaciona con el físico, porque para hacer un ejercicio responsable de la ciudadanía los individuos deben saber qué usos de la tecnología son ilegítimos y cuáles no los son. Según Cobo (2019a), el conocimiento sobre el uso de los dispositivos ya no es suficiente y el foco debe estar puesto en lo que es posible hacer con ellos, educando para un uso saludable y beneficioso de la tecnología.

Tal vez sea respecto a las redes sociales donde el foco se ha puesto con mayor énfasis en los riesgos asociados a su uso. De una entrevista exploratoria mantenida con una profesional del Plan Ceibal para la preparación del presente trabajo surge que la institución ha dedicado mucho esfuerzo a combatir el ciberbullying, el grooming o el sexting, prácticas asociadas a riesgos en términos de convivencia y privacidad. Surge, además, que aún no existe en Uruguay un marco de trabajo para la formación de ciudadanía digital, más allá del trabajo en formación de habilidades digitales críticas que lleva adelante el Plan Ceibal a través de la Red Global de Aprendizajes y las Jornadas de Ciudadanía Digital que ha comenzado a desarrollar la Agesic desde 2018.

Pero eso no basta para la formación de ciudadanos digitales. Pangrazio (2018) considera oportuno incluir la formación en el uso de las redes sociales, porque conocer su arquitectura y funcionamiento es vital para entender los riesgos, pero también las oportunidades que ofrecen.

La educación necesaria para esta nueva era digital debe centrarse en la AMI, que es aquella que procura formar a los futuros ciudadanos en el funcionamiento del nuevo espacio público virtual. Especialmente porque la forma en que la información se genera y circula en ese espacio exige otros requerimientos para su comprensión y análisis crítico. 
La AMI es un elemento clave para la participación democrática y un elemento esencial para que los ciudadanos decidan racionalmente, sean agentes de cambio y se involucren exitosamente con la información que circula. Las competencias que ofrece permiten a los nuevos ciudadanos reconocer las agendas políticas de personas y corporaciones en el nuevo espacio público, al tiempo que alzar su voz en defensa de sus propios intereses y de aquellos que no tienen una voz en este nuevo escenario (Pangrazio, 2018; Mihailidis y Thevenin, 2013). Un conocimiento más profundo del espacio público virtual permite aprovechar las nuevas reglas de juego para favorecer cambios positivos en la comunidad.

Este enfoque debe estar íntimamente vinculado con la educación cívica, pues el objetivo central es la formación de ciudadanos activos, informados y con capacidad para evaluar de forma crítica el funcionamiento del nuevo escenario (Mihailidis y Thevenin, 2013). Como explica Cobo (2019b), el cambio en ciernes hace «necesario desarrollar una nueva comprensión de lo que significa alfabetismo digital crítico, una ciudadanía digital que permita comprender y actuar frente a las nuevas reglas del juego».

Recientemente, el índice de alfabetización mediática de la Open Society Foundation (Lessenki, 2018) ha intentado demostrar cómo un sistema educativo sólido y un clima social de apertura y libertad son las mejores armas para combatir la desinformación que muchas veces se desprende de un mal uso de internet y las redes sociales. En cambio, cuando la opción es la restricción y la censura, las sociedades están peor preparadas para afrontar el problema.

Pero la AMI no debe enfocarse en políticas de contingencia contra la circulación de noticias falsas, porque estas no son más que el síntoma evidente de una enfermedad profunda. El esfuerzo de transformación educativa que se requiere implica rediseñar las políticas de educación cívica para que los ciudadanos sean capaces de manejarse con libertad y autonomía en un entorno con nuevas oportunidades y desafíos.

Sin embargo, respecto al consumo de información y noticias, la AMI debe tener un rol central en la formación de nuevos ciudadanos, porque los patrones de consumo, producción y difusión de las noticias han cambiado. Según expresa Pangrazio (2018), el consumo de noticias ya no es una cuestión exclusivamente de información, sino que, con la intermediación de las redes sociales y la forma en que las noticias pueden compartirse, el consumo de información se ha convertido en «un canal para las relaciones afectivas entre individuos» (Pangrazio, 2018).

Dado que las estructuras de estas plataformas utilizan algoritmos de personalización, se impone como una necesidad que los ciudadanos tengan conocimiento de la forma en que las plataformas funcionan y de los efectos que estas tienen sobre el espacio público (Pangrazio, 2018; Pariser, 2011). No se trata únicamente de ser capaces de identificar fuentes de información, valorar su rigurosidad y las condiciones contextuales en las cuales se emite, sino también conocer en profundidad la estructura de este nuevo 
espacio público virtual que funciona en medio de una opacidad en extremo peligrosa (Wagerif, 2018).

\section{Consideraciones finales}

El presente ensayo pretende llamar la atención sobre un tema que debería ser considerado a la hora de discutir el rol del sistema educativo en la formación de ciudadanos para el ejercicio de sus derechos y libertades en un nuevo espacio público virtual que supone nuevas reglas de juego.

Las consideraciones finales, por tanto, no pueden ser más que hipótesis generales, no solo para futuras investigaciones que permitan generar mejor evidencia empírica a nivel local (Olascoaga, 2013), sino también para el debate y la discusión de políticas públicas orientadas a resolver los problemas que enfrentarán los ciudadanos del futuro.

Esas hipótesis son tres:

a. Que el cambio en las tecnologías de la información y la comunicación supone un riesgo inédito y de grandes proporciones para los sistemas democráticos.

b. Que ese impacto se evidencia en una reestructuración del espacio público que genera dos espacios paralelos - con interacciones complejas- en los que las reglas de juego son profundamente distintas. En el espacio público virtual gobierna una situación de anomia y opacidad que debe ser discutida.

c. Que las democracias del mundo se enfrentan al dilema de contrarrestar el impacto de la tecnología a través de dos estrategias simultáneas: la regulación y las políticas educativas. Sin embargo, solo estas últimas serán capaces de afrontar los desafíos que presenta el espacio público virtual sin afectar de forma irreparable el sistema de libertades fundamentales que sustenta la estructura del sistema democrático.

\section{Bibliografía}

Balaguer, R. (2017). Vivir en la nube: Adolescencia en tiempos digitales. Montevideo: Aguilar.

Bauman, Z. y Lyon, D. (2013). Liquid surveillance: A conversation. Cambridge: Polity Press.

Bobbio, N. (1994). Liberalismo y democracia. México: Fondo de Cultura Económica. Bobbio, N. (1986). El futuro de la democracia. México: Fondo de Cultura Económica. 
Bozdag, E. y Van Den Hoven, J. (2015). Breaking the filter bubble: Democracy and design. Ethics and Information Technology, 17(4), 249-265.

Brown, C. y Czerniewicz, L. (2010). Debunking the 'digital native': Beyond digital apartheid, towards digital democracy. Journal of Computer Assisted Learning, 26(5): 357-369.

Brown, N. (2013). Egypt's Failed Transition. Journal of Democracy, 24(4), 45-58.

Chander, A. y Krishnamurthy, V. (2018). The myth of platform neutrality. Georgetown Law Technology Review, 2(2), 400-416.

Cobo, C. (2019a). Ciudadanía digital y educación: Nuevas ciudadanías para nuevos entornos. Revista Mexicana de Bachillerato a Distancia, 21(11).

Cobo, C. (2019b). Acepto las condiciones: Usos y abusos de las tecnologías digitales. Madrid: Fundación Santillana.

Digital, Culture, Media and Sports Committee (2019). Committee publishes further documents from Brittany Kaiser relating to Cambridge Analytica and Leave. EU. Recuperado de https://www.parliament.uk/business/committees/committees-a-z/ commons-select/digital-culture-media-and-sport-committee/news/brittany-kaiser-additional-papers-published/.

Deibert, R. (2019). The road to digital unfreedom: Three painful truths about social media. Journal of Democracy, 30(1), 25-39.

Dodle, M. (2018). Uruguay, sociedad e internet: Principales resultados de la encuesta WIP+DISTO Uy 2017. Montevideo: Universidad Católica del Uruguay.

Eduy21. (2018). Libro abierto: Propuestas para apoyar el acuerdo educativo. Recuperado de www.eduy21.org/libroabierto.

Floridi, L. (2019). Prólogo: El nuevo poder gris. En C. Cobo, Acepto las condiciones: Usos y abusos de las tecnologías digitales. Madrid: Fundación Santillana.

Floridi, L. (2016). Technology and democracy: Three Lessons from Brexit. Philosophy and Technology, 29(3), 189-193.

Helbing, D., Frey, B.S., Gigerenzer, G., Hafen, E., Hagner, M., Hofstetter, Y, ... Zwitter, A. (2017). Will democracy survive big data and artificial intelligence? Scientific American, 316(2). Recuperado de https://www.scientificamerican.com/article/willdemocracy-survive-big-data-and-artificial-intelligence/.

Kaiser, B. (2019). Targeted: The Cambridge Analytica whistleblower's inside story of how big data, Trump, and Facebook broke democracy and how it can happen again. Nueva York: Harper Collins.

Lyon, D. (2018). The culture of surveillance: Watching as a way of life. Cambridge: Polity Press.

Mihalialidis, P.y Thevenin, B. (2013). Media literacy as a core competency for engaged citizenship in participatory democracy. American Behavioral Scientist, 57(11), 1611-1622. 
Monhan, T. (2011). Surveillance as a cultural practice. The Sociological Quarterly, 52(4), 495-508.

Mounk, Y. (2018). The people vs. Democracy: why our freedom is in danger and how to save it. Cambridge, MA: Harvard University Press.

Mossberger, K. (2009). Toward digital citizenship: Addressing inequality in the information age. En A. Chadwick y P. Howard (eds.), Routledge handbook of internet politics (pp. 173-185). Nueva York: Taylor \& Francis.

Newman, N., Fletcher, R., Kalogeropoulos, A. y Nielsen, R. K. (2019). Digital News Report 2019. Recuperado de http://www.digitalnewsreport.org/.

O'Neil, C. (2016). Weapons of math destruction: How big data increases inequality and threatens democracy. Nueva York: Crow.

Olascoaga, N. y García, S. (2013). Alfabetización informacional en Uruguay: ¿dónde estábamos y dónde estamos? Informatio, 18(2),77-102.

Lessenki, M. (2018). Common sense wanted: Resilience to "post-truth» and its predictors in the new media literacy index. Sofía: OSF.

Pangrazio, L. (2018). What's new about «fake news»?: Critical digital literacies in an era of fake news, post-truth and clickbait. Páginas de Educación, 11 (1), 6-22.

Papacharissi, Z. (2008). The virtual sphere 2.0: The internet, the public sphere, and beyond. En A. Chadwick y P. Howard (eds.), Routledge Handbook of Internet Politics (pp. 230-245). Nueva York: Taylor \& Francis.

Pariser, E. (2011). Filter bubble: What the internet is hiding to us. Nueva York: The Penguin Press.

Peirano, M. (2019). El enemigo conoce el sistema: Manipulación de ideas, personas e influencias después de la economía de la atención. Madrid: Debate.

Persily, N. (2017). Can Democracy Survive the Internet? Journal of Democracy, 28(2), 63-76.

Pew Research Center. (2018). News use across social media platforms 2018. Recuperado de https://www.journalism.org/2018/09/10/news-use-across-social-mediaplatforms-2018/

Putnam, R. (2000). Bowling alone: The colapse and revival of American community. Nueva York: Simon \& Shuster.

Radar. (2018). El perfil del internauta uruguayo. Recuperado de http://www.gruporadar.com.uy/01/16-12-2018-presentamos-la-15a-edicion-del-perfil-del-internauta-uruguayo-2018/?lang=en.

Rawls, J. (1995). Liberalismo político. México: Fondo de Cultura Económica.

Richards, N. M. (2013). The dangers of surveillance. Harvard Law Review, 126(7), 19341965. Recuperado de https://www.jstor.org/stable/23415062.

Sunstein, C. (2016). \#Republic: Divided democracy in the age of social media. Princeton: Princeton University Press. 
Schwab, K. (2016). The fourth industrial revolution. Nueva York: Crown Bussines.

Shahbaz, A. y Funk, A. (2019). The crisis of social media: Freedom house. Recuperado de https://www.freedomonthenet.org/sites/default/files/2019-11/11042019_Report_FH_FOTN_2019_final_Public_Download.pdf.

Thaler, R. y Sunstein, C. (2003). Libertarian paternalism. Behavioral Economics, Public Policy, and Paternalism, 93(2), 175-179.

Thaler, R. y Sunstein, C. (2008). Nudge: Improving decisions about health, wealth, and happiness. New Haven: Yale University Press.

Toffler, A. (1992). El cambio de poder. Barcelona: Plaza \& Janés.

Wilson, C. (2012). Alfabetización mediática e informacional: proyecciones didácticas. Comunicar, 39(20), 15-24.

Walker, C. (2018). What is sharp power? Journal of Democracy, 29(3), 9-23.

Wegerif, R. (2018). New technology and the apparent failure of democracy: An educational response. On Education: Journal for Research and Debate, 1. (1).

Youmans, W. y York, J. (2012). Social media and the activist toolkit: User agreements, corporate interests, and the information infrastructure of modern social movements. Journal of Communication, 62, 315-329.

Zuboff, S. (2015). Big other: Surveillance capitalism and the prospects of an information civilization. Journal of Information Technology, 30, 7589.

Zuboff, S. (2019). The age of surveillance capitalism: The fight for a human future at the new frontier of power. Nueva York: Public Affairs. 